\title{
Perspectives on beam-shaping optimization for thermal-noise reduction in advanced gravitational-wave interferometric detectors: Bounds, profiles, and critical parameters
}

\author{
Vincenzo Pierro, Vincenzo Galdi, ${ }^{* \dagger}$ Giuseppe Castaldi, and Innocenzo M. Pinto \\ Waves Group, Department of Engineering, University of Sannio, I-82100 Benevento, Italy \\ Juri Agresti and Riccardo DeSalvo \\ LIGO Laboratory, California Institute of Technology, Pasadena, California 91125, USA
}

(Received 4 July 2007; published 18 December 2007)

\begin{abstract}
Suitable shaping (in particular, flattening and broadening) of the laser beam has recently been proposed as an effective device to reduce internal (mirror) thermal noise in advanced gravitational-wave interferometric detectors. Based on some recently published analytic approximations (valid in the infinite-testmass limit) for the Brownian and thermoelastic mirror noises in the presence of arbitrary-shaped beams, this paper addresses certain preliminary issues related to the optimal beam-shaping problem. In particular, with specific reference to the Laser Interferometer Gravitational-wave Observatory (LIGO) experiment, absolute and realistic lower bounds for the various thermal-noise constituents are obtained and compared with the current status (Gaussian beams) and trends (mesa beams), indicating fairly ample margins for further reduction. In this framework, the effective dimension of the related optimization problem, and its relationship to the critical design parameters are identified, physical-feasibility and model-consistency issues are considered, and possible additional requirements and/or prior information exploitable to drive the subsequent optimization process are highlighted.
\end{abstract}

DOI: 10.1103/PhysRevD.76.122003

PACS numbers: 04.80.Nn, 07.60.Ly, 41.85.Ct, 42.55.-f

\section{INTRODUCTION}

In all currently operating (and possibly future) interferometric gravitational-wave detectors, the overall limit sensitivity of the instrument is bounded by the noise floor, which, in the most interesting observational frequency band $(30-300 \mathrm{~Hz})$, is dominated by thermal noises in the substrate and in the high-reflectivity coating of the test masses. With particular reference to the Laser Interferometer Gravitational-wave Observatory (LIGO) experiment [1], an introductory discussion of the various noise components can be found in [2], and a numerical code for computing the noise budget is available from [3]. Toward the development of second-generation detectors, such as Adv-LIGO [4], the quest for increasing the event rate in the observational band has motivated the exploration of various techniques for reducing the mirror thermal noise. With specific reference to the coating Brownian noise (dominant in the current baseline design featuring fused-silica test masses), use of improved (low-mechanical-loss) materials [5], geometric optimization of the coating design [6], and flat-top (commonly referred to as "mesa") beams [7,8] seem the most promising. The latter option, intuitively motivated by the potential capability of a mesa beam (MB) of better averaging the thermally induced mirror surface fluctuations as compared to a standard Gaussian beam (GB), has been numerically proved to yield significant reductions in the overall thermal noise $[9,10]$ and has

\footnotetext{
*vgaldi@unisannio.it

${ }^{\dagger}$ http://www.ing.unisannio.it/vgaldi
}

led to the development of a cavity prototype with nonspherical "Mexican hat" (MH) profile mirrors [11,12]. Alternative (nearly concentric [13], nearly spheroidal [14-16]) designs have been subsequently proposed to cope with the inherent tilt-instability of the originally conceived nearly flat configuration. Also, use of higherorder modes in standard spherical cavities has been shown to provide, in principle, comparable reductions without the need of changing the mirror profile [17], but its practical feasibility still remains to be assessed.

The method utilized in [10] to compute the coating and substrate thermal noises for arbitrary-shaped beams relies on a finite test mass (FTM) computationally intensive numerical analysis based on the approach in $[18,19]$. More recently [20,21], a simple analytic approximation has been derived in the infinite-test-mass (ITM) limit, based on the approach in [22]. This approximation has been validated and calibrated in [21] against the FTM numerical solutions (see also the discussion in Sec. III B 3). In view of its remarkably simple form, in terms of spectral integral functionals of the beam intensity profile, it appears suggestive to exploit it for addressing the optimal beam-shaping problem, i.e., finding the beam profile that minimizes a given thermal-noise constituent. In a step-by-step approach, acknowledging the formal and computational complexity of the arising optimization problem, this paper addresses some key preliminary issues. In particular, emphasis is placed on the a priori deduction of absolute and realistic lower bounds for the various thermal-noise constituents, the identification of the effective dimension of the problem, and how this depends on the 
critical design parameters, and the gathering of additional requirements and/or prior information to be utilized in the actual optimization problem.

Accordingly, this paper is organized as follows. In Sec. II, the problem geometry, formulation, notation, and strategy are outlined, with a compact review of the relevant background theory (ITM approximation). In Sec. III, under the idealized assumption of zero diffraction-loss (compact spatial support) beam profiles, absolute lower bounds for the noise constituents, as well as the corresponding beam profiles over the mirror, are obtained in analytic form, by solving a straightforward variational problem. Subsequently, a key physical feasibility constraint (related to the finite-spatial bandwidth of the cavity eigenmodes) is taken into account by approximating the above compactsupport optimal profiles in a suitable $L^{2}$ functional subspace, whose dimension is fixed by the diffraction-loss constraint. This results in more realistic tighter bounds. In this framework, the role of the number of electromagnetic degrees of freedom [23] of the cavity in setting the effective dimension of the optimization problem is highlighted. Moreover, some model-consistency issues are discussed in order to assess the practical relevance of the results. In Sec. IV, the obtained absolute and realistic lower bounds for the considered noise components are compared to the levels currently achievable using GB and MB profiles. Finally, in Sec. V, conclusions and recommendations are provided.

\section{PROBLEM STATEMENT}

\section{A. Geometry}

Referring to the problem geometry illustrated in Fig. 1, we consider a standard Fabry-Perot optical cavity with two identical, symmetric, nearly flat (nonspherical) mirrors of radius $a$ laid on cylindrical test masses, separated by a distance $L$ [see Fig. 1(a)]. The mirror (axisymmetric) departure from flatness is described by $h(r)$, with $r$ denoting the radial coordinate in the mirror plane [see Fig. 1(b)]. In what follows, attention is focused on the axisymmetric (i.e., $\theta$-independent) eigenmode field distribution $\Phi(r)$ on the mirror, with implicit $\exp (\iota \omega t)$ time-harmonic depen- (a)

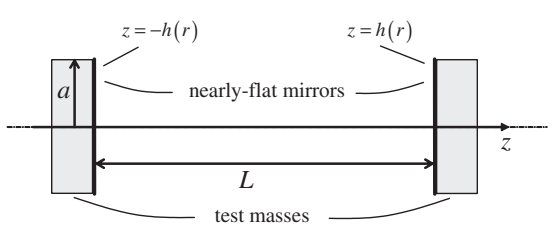

(b)



FIG. 1. Problem schematic: A perfectly symmetric FabryPerot optical cavity composed of two nearly flat mirrors [with profile $h(r)$ ] attached on cylindrical test masses of radius a separated by a distance $L$ along the $z$ axis. (a): Side view. (b): Front view. dence. Note that, in view of the duality relations expounded in [24], the results derived hereafter apply to the nearly concentric case too [25].

\section{B. Background: Infinite-test-mass approximations}

In the ITM approximation [20,21], and in the low frequency limit of interest for gravitational-wave interferometers, the power spectral densities of the main coating and substrate thermal-noise constituents of interest can be written as

$$
S=C \int_{0}^{\infty} \kappa^{q+1}\left\{\mathcal{H}\left[|\Phi|^{2}\right](\kappa)\right\}^{2} d \kappa,
$$

where $C$ is a noise-type- and frequency-dependent factor (irrelevant for all further developments), $q$ is a noise-typedependent scaling exponent (see Table I), $\Phi(r)$ is the axisymmetric eigenmode field distribution on the mirror, and

$$
\mathcal{H}[F](\xi) \equiv \int_{0}^{\infty} F(\zeta) J_{0}(\xi \zeta) \zeta d \zeta
$$

denotes the Hankel-transform (HT) operator. Here and henceforth, $J_{m}$ denotes an $m$ th-order Bessel function of the first kind ([26], Sec. 9.1). The (axisymmetric) field distribution $\Phi(r)$ satisfies the eigenvalue equation [27]

$$
\gamma \Phi(r)=\int_{0}^{a} K\left(r, r^{\prime}\right) \Phi\left(r^{\prime}\right) r^{\prime} d r^{\prime},
$$

where $\gamma$ denotes the half-roundtrip eigenvalue, and the kernel is given by

$$
\begin{aligned}
K\left(r, r^{\prime}\right)= & \frac{l k}{L} J_{0}\left(\frac{k r r^{\prime}}{L}\right) \exp (-\imath k L) \\
& \times \exp \left\{\imath k\left[h(r)+h\left(r^{\prime}\right)-\frac{\left(r^{2}+r^{\prime 2}\right)}{2 L}\right]\right\},
\end{aligned}
$$

with $k=2 \pi / \lambda$ denoting the free-space wavenumber $(\lambda$ being the wavelength). Equations (3) and (4) can be recognized as a mapping between a mirror profile $h(r)$ and a set $\Omega(h)=\left\{\left[\gamma_{m}, \Phi_{m}\right], m=1,2, \ldots\right\}$ of eigenstates. Here and henceforth, unless otherwise specified, the field distribution on the mirror is assumed to be normalized as follows:

$$
\int_{0}^{\infty}|\Phi(r)|^{2} r d r=1
$$

In addition, a further constraint has to be enforced on the

TABLE I. Thermal-noise constituents of interest and corresponding scaling exponents [cf. (1)].

\begin{tabular}{ll}
\hline \hline \multicolumn{1}{c}{ Noise type } & $q$ \\
\hline Substrate Brownian & -1 \\
Substrate thermoelastic & 1 \\
Coating Brownian and thermoelastic & 0 \\
\hline \hline
\end{tabular}


per-mirror diffraction loss [27]

$$
\mathcal{L}[\Phi] \equiv \int_{a}^{\infty}|\Phi(r)|^{2} r d r=1-|\gamma|^{2} \leq \mathcal{L}_{T},
$$

with $\mathcal{L}_{T}$ denoting a design limiting value. For Adv-LIGO, the reference figure is $\mathcal{L}_{T}=1 \mathrm{ppm}\left(10^{-6}\right)$. The diffraction-loss constraint singles out a subset $\Omega_{C}(h) \subset$ $\Omega(h)$ of admissible eigenmodes.

\section{Formulation and notation}

It is expedient to recast the problem into a canonical form by introducing the scaled variables

$$
\bar{r}=\frac{r}{a}, \quad \bar{\kappa}=a \kappa,
$$

and the scaled field distribution

$$
\phi(\bar{r})=a \Phi(\bar{r} a) .
$$

Here and henceforth, the overbar denotes scaled quantities. The noise functional in (1) can accordingly be rewritten as

$$
S=\frac{C}{a^{q+2}} \bar{S}\left[|\phi|^{2}, q\right]
$$

where

$$
\bar{S}\left[|\phi|^{2}, q\right]=\int_{0}^{\infty} \bar{\kappa}^{q+1}\left\{\mathcal{H}\left[|\phi|^{2}\right](\bar{\kappa})\right\}^{2} d \bar{\kappa},
$$

thereby explicitly factoring out the $a^{-(q+2)}$ scaling law predicted by the ITM approximation [20,21]. In what follows, we focus on the scaled noise functional in (10), which essentially accounts for the beam-shaping effects. Unless strictly needed, the explicit dependence on $|\phi|^{2}$ and $q$ will be omitted for simplicity of notation. The scaled field distribution $\phi(\bar{r})$ in (8) satisfies the scaled version of the eigenproblem in (3), which can be conveniently recast as

$$
\bar{\gamma} \phi(\bar{r})={ }_{\imath} \pi N_{D} \exp [-\imath V(\bar{r})] \mathcal{H}_{1}[\exp (-\imath V) \phi]\left(\pi N_{D} \bar{r}\right),
$$

where $\bar{\gamma}=\gamma \exp (\imath k L)$,

$$
\mathcal{H}_{1}[F](\xi) \equiv \int_{0}^{1} F(\zeta) J_{0}(\xi \zeta) \zeta d \zeta
$$

denotes the $[0,1]$ interval-windowed HT operator, and

$$
V(\bar{r})=k h(a \bar{r})-\frac{\pi N_{D} \bar{r}^{2}}{2}
$$

is a mirror-profile-dependent phase function, with

$$
N_{D}=\frac{2 a^{2}}{\lambda L}=2 N_{F}
$$

denoting the so-called number of electromagnetic degrees of freedom $[23,28]$. This parameter, whose relevance will be illustrated later on (see Sec. III B), is strictly related to the Fresnel number $N_{F}$ of the optical cavity [27]. In the following we shall always assume the eigenfunctions as normalized, viz.,

$$
\|\phi\| \equiv\left[\int_{0}^{\infty}|\phi(\bar{r})|^{2} \bar{r} d \bar{r}\right]^{1 / 2}=1,
$$

with $\|\cdot\|$ denoting the usual $L_{[0, \infty[}^{2}$ (cylindrical) Hilbert norm. Accordingly, we shall write the diffraction-loss constraint as

$$
\mathcal{L}[\phi]=\int_{1}^{\infty}|\phi(\bar{r})|^{2} \bar{r} d \bar{r}=1-|\bar{\gamma}|^{2} \leq \mathcal{L}_{T} .
$$

\section{The optimization problem}

The optimization problem of interest consists of minimizing the scaled noise functional in (10), acting on the mirror profile $h(\bar{r})$, i.e., in finding the special mirror profile $h(\bar{r})$ (within a suitable functional class, e.g., $C^{\infty}$ ) for which

$$
\min _{\phi \in \Omega_{C}(h)}\left\|\bar{\kappa}^{q / 2} \mathcal{H}\left[|\phi|^{2}\right]\right\|^{2}
$$

takes on its smallest value, $\Omega_{C}(h)$ denoting the subset of eigenmodes obeying the diffraction-loss constraint (16). The minimization of (17), subject to (16), represents a formidable optimization problem, whose well-posedness (i.e., existence and uniqueness of the solution and its continuous dependence on data) cannot be taken for granted, with the consequent ill-conditioning problems that may arise in the numerical implementation. A further complication is posed by the general nonconvexity of the problem, which may result in multiple local minima that may trap standard descent-based optimization techniques (e.g., conjugate gradient [29]) into false solutions. Therefore, global optimization techniques need to be applied, such as genetic [30], evolutionary [31], or particleswarm [32] algorithms, whose convergence is typically rather slow. Taking into account that each iteration in the optimization procedure may require several numerical solutions of the eigenproblem in (11), the resulting overall computational burden can become prohibitive. From the above considerations, it is clear that any blind attempt of attacking such a complex and computationally demanding problem may be deemed to failure. In a step-by-step approach, it appears more reasonable to start addressing some preliminary issues, such as

(i) A priori estimation of realistic lower bounds for the various noise constituents, and comparison with the current status and trends, in order to assess the potential reduction achievable by further optimization (and, hence, its worthiness).

(ii) Identification of the effective problem's dimension, as a function of the key cavity design parameters.

(iii) Gathering of prior information (e.g., optimal beam profiles and associated structural features) to be exploited in order to intelligently drive the optimization process. 
The rest of the present paper accordingly deals with the above issues.

\section{SOME THEORETICAL BOUNDS}

\section{A. Absolute bounds: Compact-support beams}

The simplest and crudest approximation of the original optimization problem in (17), leading to a well-posed and analytically treatable problem, consists of assuming the beam profile to exhibit a compact spatial support within the $[0,1]$ interval, thereby implying zero diffraction losses. As we shall see, this implicitly prevents the profile from being a solution of the eigenproblem in (11). Letting $f \equiv|\phi|^{2}$, one is thus led to the variational problem in the space $L_{[0,1]}^{1}$ of summable functions,

$$
\bar{S}_{\mathrm{abs}}^{(\min )} \equiv \min _{f \in L_{[0,1]}^{1}}\left\|\bar{\kappa}^{q / 2} \mathcal{H}_{1}[f]\right\|^{2},
$$

under the constraints

$$
\begin{aligned}
f:[0,1] & \rightarrow \mathbb{R}^{+}, \\
\int_{0}^{1} f(\bar{r}) \bar{r} d \bar{r} & =1,
\end{aligned}
$$

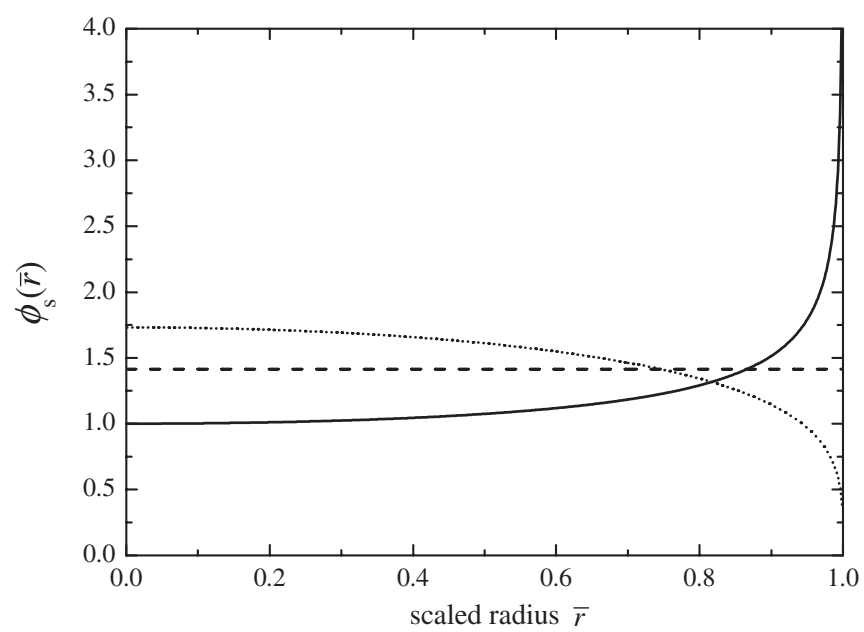

FIG. 2. Optimal (minimum-noise) compact-support beam profiles in (21). Continuous curve: substrate Brownian $(q=-1)$; dashed curve: coating $(q=0)$; dotted curve: substrate thermoelastic $(q=1)$. whose solution is given below. The arising results are anticipated to provide absolute lower bounds, which may not be attainable, in view of the mentioned unphysical simplifying assumptions. From the Lagrange theory of constrained optimization [33], the constrained variational problem in (18) and (19b) can be recast into the unconstrained optimization of the Lagrangian functional [34]

$$
\Lambda[f, \mu]=\left\|\bar{\kappa}^{q / 2} \mathcal{H}_{1}[f]\right\|^{2}-2 \mu\left[\int_{0}^{1} f(\bar{r}) \bar{r} d \bar{r}-1\right],
$$

where $\mu$ is the so-called Lagrange multiplier. It is shown in the Appendix that this problem admits a unique solution $f_{s}$ (i.e., an absolute minimum), obtainable using variational calculus, viz.,

$f_{s}(\bar{r})=\left|\phi_{s}(\bar{r})\right|^{2}=(q+2)\left(1-\bar{r}^{2}\right)^{q / 2}, \quad-1 \leq q \leq 1$,

which also satisfies the positivity constraint in (19a). The corresponding (minimum) noise components are given by

$$
\bar{S}_{\mathrm{abs}}^{(\min )}=2^{q+1} \Gamma\left(\frac{q}{2}+1\right) \Gamma\left(\frac{q}{2}+2\right),
$$

with $\Gamma$ denoting the Gamma (factorial) function ([26], Sec. 6.1). The optimal beam profiles are shown in Fig. 2, whereas the corresponding (minimum) noise values are collected in Table II.

The following remarks are in order:

(i) The noise-minimizing beam profiles can exhibit step discontinuities, or even singularities at $\bar{r}=1$ (see Fig. 2). This is neither surprising (in view of the relaxation of the physical feasibility constraints) nor undermining of the meaningfulness of the preliminary results derived at this stage as (anticipated) absolute lower bounds for the actual problem. The reader is referred to Sec. III B below for more realistic bounds.

(ii) For the coating noises $(q=0)$, the optimal profile is perfectly flat, thereby supporting previous intuitive arguments in favor of flat-top beams $[7,8]$.

(iii) For the substrate noises, the optimal beam profile is appreciably rounded (nonflat) for the thermoelastic component $(q=1)$. This should be taken into account when assessing the performance of configu-

TABLE II. Comparison between absolute [cf. (22)] and realistic (for $a=16 \mathrm{~cm}$, i.e., $N_{D}=$ 12.03, extracted from Fig. 6) noise bounds. Also shown, as references, are the noise levels attainable with $\mathrm{GB}$ and reference $\mathrm{MB}$ (minimum noise, for $a=16 \mathrm{~cm}$, i.e., $N_{D}=12.03$, cf. Figure 9) profiles.

\begin{tabular}{llccccc}
\hline \hline$q$ & \multicolumn{1}{c}{$\bar{S}_{\mathrm{abs}}^{(\min )}$} & $\bar{S}_{\mathrm{BL}} / \bar{S}_{\mathrm{abs}}^{(\min )}$ & $\bar{S}_{\mathrm{GB}} / \bar{S}_{\mathrm{abs}}^{(\min )}$ & $\bar{S}_{\mathrm{MB}}^{(\min )} / \bar{S}_{\mathrm{abs}}^{(\min )}$ & $\bar{S}_{\mathrm{GB}} / \bar{S}_{\mathrm{BL}}$ & $\bar{S}_{\mathrm{MB}}^{(\min )} / \bar{S}_{\mathrm{BL}}$ \\
\hline-1 & 1.5708 & 1.145 & 2.965 & 2.043 & 2.591 & 1.785 \\
0 & 2 & 1.313 & 6.907 & 3.238 & 5.256 & 2.465 \\
1 & 4.712 & 1.552 & 13.658 & 4.454 & 8.801 & 2.870 \\
\hline \hline
\end{tabular}


rations featuring sapphire test masses, for which substrate thermoelastic noise is known to be dominant [10]. In this framework, use of hyperboloidal beams [14-16] as physically feasible approximants should be explored. On the other hand, the optimal intensity profile for the Brownian component $(q=$ -1 ) is close to flat, with a steep increase at the mirror's edge. This is clearly unphysical, but may be suggestive of using an annular beam. Note that the above results pertain to the minimization of a single noise constituent. Extensions to the minimization of a given combination of noise constituents are possible, but most likely need to be pursued numerically, via suitable discretization of the involved operators.

\section{B. More realistic (tighter) bounds: Diffraction-loss vs band-limitation constraints}

Besides the diffraction-loss constraint, a less obvious (and competing) constraint exists, stemming from an abstract property of the eigenmodes of (11): band limitation.

\section{Band-limitation property}

Applying the HT operator at both sides of the eigenproblem in (11) and using the more or less obvious identities

$$
\mathcal{H}_{1}[f]=\mathcal{H}[\Pi(\bar{r}) f(\bar{r})], \quad \mathcal{H}[\mathcal{H}[f]]=f,
$$

with $\Pi$ denoting the unit rectangular-window function, $\Pi(\xi)=1,0 \leq \xi \leq 1, \Pi(\xi)=0, \xi>1$, one obtains

$$
\mathcal{H}[\phi \exp (\imath V)]\left(\pi N_{D} \bar{r}\right)={ }_{\imath} \frac{\pi N_{D}}{\bar{\gamma}} \Pi(\bar{r}) \exp \left[-{ }_{\imath} V(\bar{r})\right] \phi(\bar{r}) \text {. }
$$

Equation (24) shows that the HT of the function $\phi \exp (l V)$ (and, a-fortiori, of the function $\phi$ ) has a compact support, vanishing outside $\left[0, \pi N_{D}\right]$. Technically, the HT plays the role of a wavenumber spectrum, and accordingly $\pi N_{D}$ is the spatial bandwidth of the field. Note that the spatial bandwidth is proportional to the number of electromagnetic degrees of freedom $N_{D}$ in (14). It is therefore natural to try approximating the optimal (but, as anticipated, unphysical) beam profiles obtained in Sec. III A using a basis in $L_{[0, \infty[}^{2}$ with finite-spatial bandwidth $\pi N_{D}$. It is worth stressing that no constructive procedure is given for retrieving a mirror profile for which such a superposition is an actual eigenmode. Nonetheless, being a physically admissible (finite-spatial bandwidth) profile, it is expected to yield tighter noise bounds, as compared to (22).

\section{Prolate-spheroidal wave-function expansion}

A more or less obvious choice for the space-bandlimited basis is provided by the so-called prolatespheroidal wave-functions (PSWFs) [35-38], which satisfy the eigenproblem [39]

$$
\bar{\eta} \varphi(\bar{r})={ }_{\imath} \pi N_{D} \mathcal{H}_{1}[\varphi]\left(\pi N_{D} \bar{r}\right) .
$$

In our implementation, the PSWFs are calculated following the approach in [37]. It can be shown that (apart from irrelevant complex multiplicative constants) the solutions of (25), $\varphi_{n}$, are real and satisfy the double-orthogonality condition

$$
\left\langle\varphi_{n}, \varphi_{m}\right\rangle=\delta_{n m}, \quad\left\langle\varphi_{n}, \varphi_{m}\right\rangle_{1}=\bar{\eta}_{n} \delta_{n m},
$$

where $\delta_{m n}$ is the Kronecker symbol, $\bar{\eta}_{n}$ indicates the $n$th eigenvalue of (25), and $\langle\cdot, \cdot\rangle$ and $\langle\cdot, \cdot\rangle_{1}$ denote the $L_{[0, \infty[}^{2}$ and $L_{[0,1]}^{2}$ (cylindrical) inner product, respectively. The eigenvalue spectrum of (25), shown in Fig. 3 for several values of $N_{D}$, has a steplike behavior: the first $\sim N_{D}$ eigenvalues are close to 1 in magnitude, while the remaining decay exponentially to zero [36]. The semilog scale utilized in Fig. 3 highlights the steplike behavior (with exponentially decaying tail) of the eigenvalue spectrum. The doubleorthogonality condition in (26) implies

$$
\int_{1}^{\infty}\left|\varphi_{m}(\bar{r})\right|^{2} \bar{r} d \bar{r}=1-\left|\bar{\eta}_{m}\right|^{2}
$$

In view of (27) and the noted behavior of the eigenvalues, the first $\sim N_{D}$ eigenmodes are almost fully localized in $[0,1]$, while the remaining ones are almost fully (de)localized to $\bar{r}>1$. A plot of a few PSWFs of increasing order is shown in Fig. 4, for $N_{D}=12$ (corresponding, for $L=$ $4 \mathrm{~km}$ and $\lambda=1064 \mathrm{~nm}$, to a mirror radius $a \approx 16 \mathrm{~cm}$ of interest for Adv-LIGO). Also shown, as a reference, is the behavior of the infinite-mirror (LG-type) solutions. It is observed that the agreement between the two is rather good

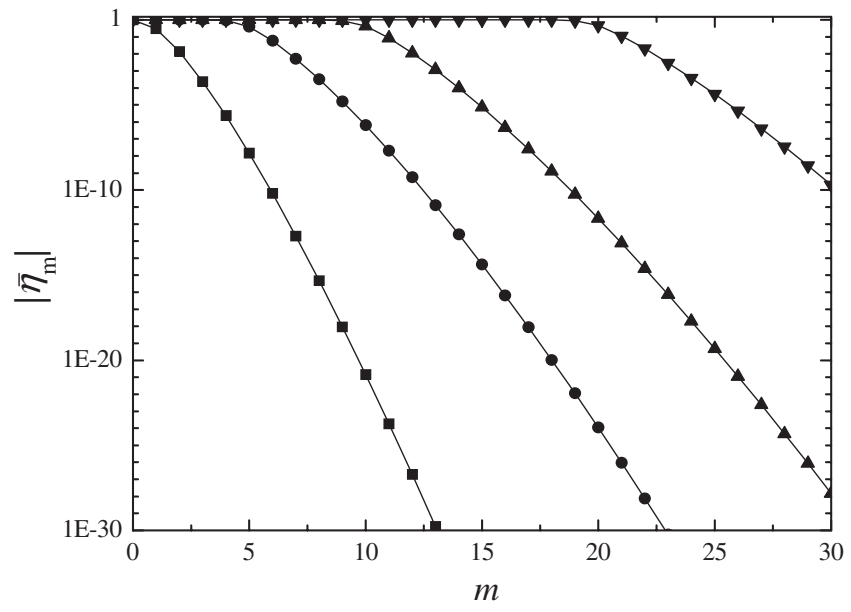

FIG. 3. PSWF eigenvalues (magnitude) as a function of order $m$, for various values of $N_{D}$. The semilog scale highlights the step behavior with exponential tail (see the discussion in Sec. III B 2). Squares: $N_{D}=1$; circles: $N_{D}=5$; up-triangles: $N_{D}=10$; down-triangles: $N_{D}=20$. 

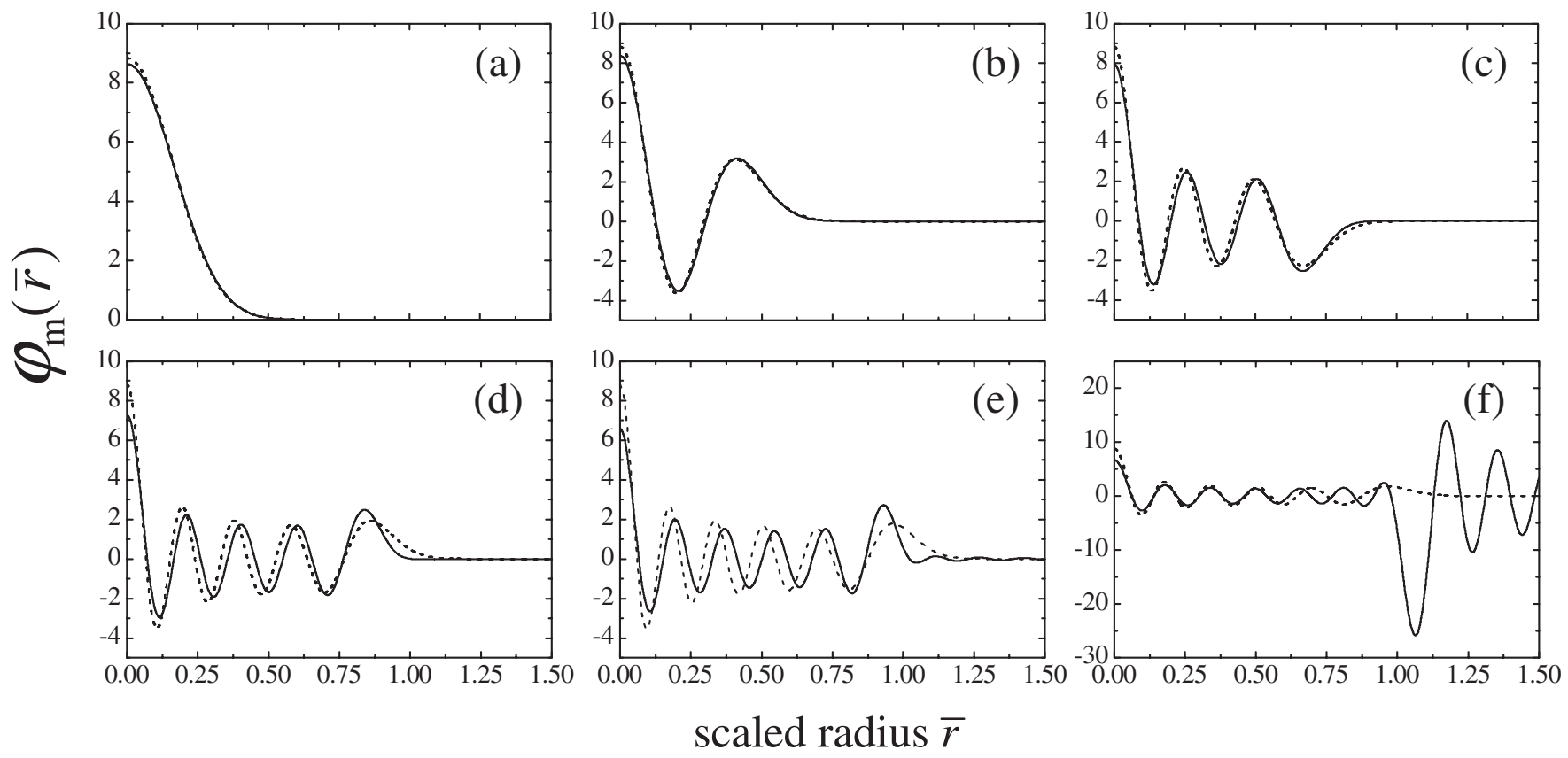

FIG. 4. PSWF profiles for $N_{D}=12$ and various orders (solid curves). (a): $m=0$; (b): $m=2 ;$ (c): $m=5 ;(\mathrm{d}): m=8 ;(\mathrm{e}): m=10$; (f): $m=13$. Also shown, as reference (dashed curves), is the behavior of the infinite-mirror (LG-type) solutions.

for low orders, for which the functions are localized, and deteriorates as the order $m$ approaches $N_{D}$, beyond which the functions exhibit the anticipated delocalization.

The best (in $L^{2}$ norm) band-limited approximation of the compact-support minimum-noise beam profiles is therefore provided by the PSWF expansion

$$
\phi_{\mathrm{BL}}(\bar{r})=\sum_{m=0}^{M_{T}-1} c_{m} \varphi_{m}(\bar{r}),
$$

with the coefficients $c_{m}$ obtained via Fourier-type projection [40],

$$
c_{m}=\frac{\left\langle\phi_{s}, \varphi_{m}\right\rangle_{1}}{\sqrt{\sum_{n=0}^{M_{T}-1}\left(\left\langle\phi_{s}, \varphi_{n}\right\rangle_{1}\right)^{2}}} .
$$

It is readily shown that the truncation order $M_{T}$ in (28) is dictated by the prescribed diffraction loss. Under the ideal steplike assumption for the eigenvalue dependence on index, whereby $\left|\bar{\eta}_{m}\right|=1, \forall m \leq N_{D}$ and $\bar{\eta}_{m}=0, \forall m>$ $N_{D}$, the diffraction-loss constraint would be satisfied for any $M_{T} \leq N_{D}$, however small the prescribed $\mathcal{L}_{T}$. A conservative estimate of $M_{T}$, taking into account the actual, albeit tiny, departure of the $m<N_{D}$ eigenvalue magnitudes from unity may be obtained from the obvious inequality

$$
\begin{aligned}
\mathcal{L}\left[\phi_{\mathrm{BL}}\right] & =\sum_{m=0}^{M_{T}-1}\left(1-\left|\bar{\eta}_{m}\right|^{2}\right)\left|c_{m}\right|^{2} \\
& \leq\left(1-\left|\bar{\eta}_{M_{T}-1}\right|^{2}\right) \sum_{m=0}^{M_{T}-1}\left|c_{m}\right|^{2}=\left(1-\left|\bar{\eta}_{M_{T}-1}\right|^{2}\right),
\end{aligned}
$$

where use has been made of the double-orthogonality conditions in (26), the fact that the $\left|\bar{\eta}_{m}\right|$ form a monotonically decreasing sequence, and the unit-norm constraint, viz.

$$
\sum_{m=0}^{M_{T}-1}\left|c_{m}\right|^{2}=\left\|\phi_{\mathrm{BL}}\right\|^{2}=1 .
$$

We accordingly get the following (conservative) estimate for the truncation order, which sets the effective dimension (number of available design parameters) of the beam (mirror) optimization problem:

$$
M_{T}=\text { largest } m:\left(1-\left|\bar{\eta}_{m-1}\right|^{2}\right) \leq \mathcal{L}_{T} .
$$

For $\mathcal{L}_{T}=1 \mathrm{ppm}$, the truncation index $M_{T}$ computed from (30) is plotted as a function of $N_{D}$ in Fig. 5. We may loosely conclude that the effective dimension of the optimization problem is

$$
M_{T} \lesssim N_{D}
$$

The inequality in (30) will be reasonably tight when representing functions that are essentially localized within the unit-disc (mirror-confined beams), whose projection onto 


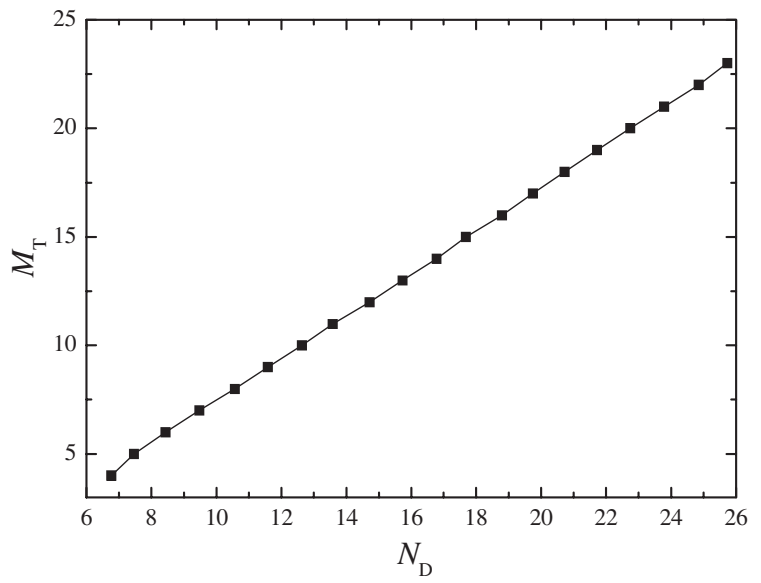

FIG. 5. Truncation index $M_{T}$ in the PSWF expansion [cf. (32)] as a function of number of electromagnetic degrees of freedom $N_{D}$.

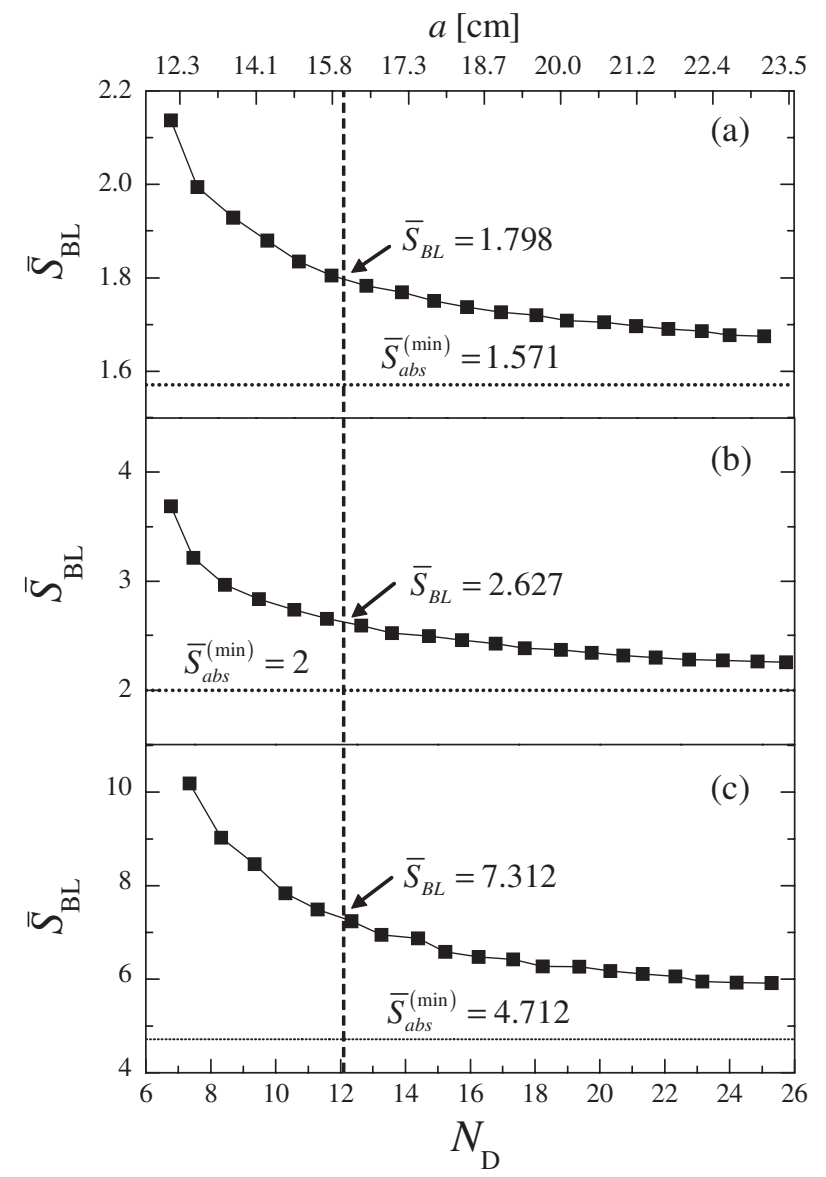

FIG. 6. Realistic noise bounds obtained from band-limited beam profiles [cf. (28)] and truncation index $M_{T}$ as in (32) [cf. Fig. 5], as a function of $N_{D}$. (a): Substrate Brownian $(q=$ $-1)$; (b): Coating $(q=0)$; (c): Substrate thermoelastic $(q=1)$. Also shown, as references, are the corresponding mirror-radius scale (top axis, assuming $L=4 \mathrm{~km}$ and $\lambda=1064 \mathrm{~nm}$ ), the absolute bounds [dotted lines, cf. (22)], and the noise values for $a=16 \mathrm{~cm}$ (i.e., $N_{D}=12.03$ ). the delocalized eigenstates with $m \gtrsim N_{D}$ is negligibly small.

More or less obviously, the accuracy of (28) is strictly dependent on (and expected to increase with) the number of terms in the truncated expansion. It therefore makes sense to check how close one can get to the optimal profiles for various meaningful values of $N_{D}$ [and hence, via (32), $\left.M_{T}\right]$.

Figures 6(a)-6(c) show the behavior of the noises associated with the band-limited profiles in (28), referred to as $\bar{S}_{\mathrm{BL}}$, as a function of $N_{D}$. For all three cases, the noise decreases with increasing $N_{D}$, and appears to asymptotically approach values close to the absolute bounds in (22). Recalling (14), the variation of $N_{D}$ was obtained by tuning the cavity length $L$ and the laser wavelength $\lambda$ at the reference values in Adv-LIGO, and taking the mirror radius $a$ within a realistic range (see the scale on the top axis of Fig. 6). For each value of $a$ (and, hence, $N_{D}$ ), the truncation index $M_{T}$ (cf. Fig. 5) was derived according to (32), with $\mathcal{L}_{T}=1 \mathrm{ppm}$ [41].

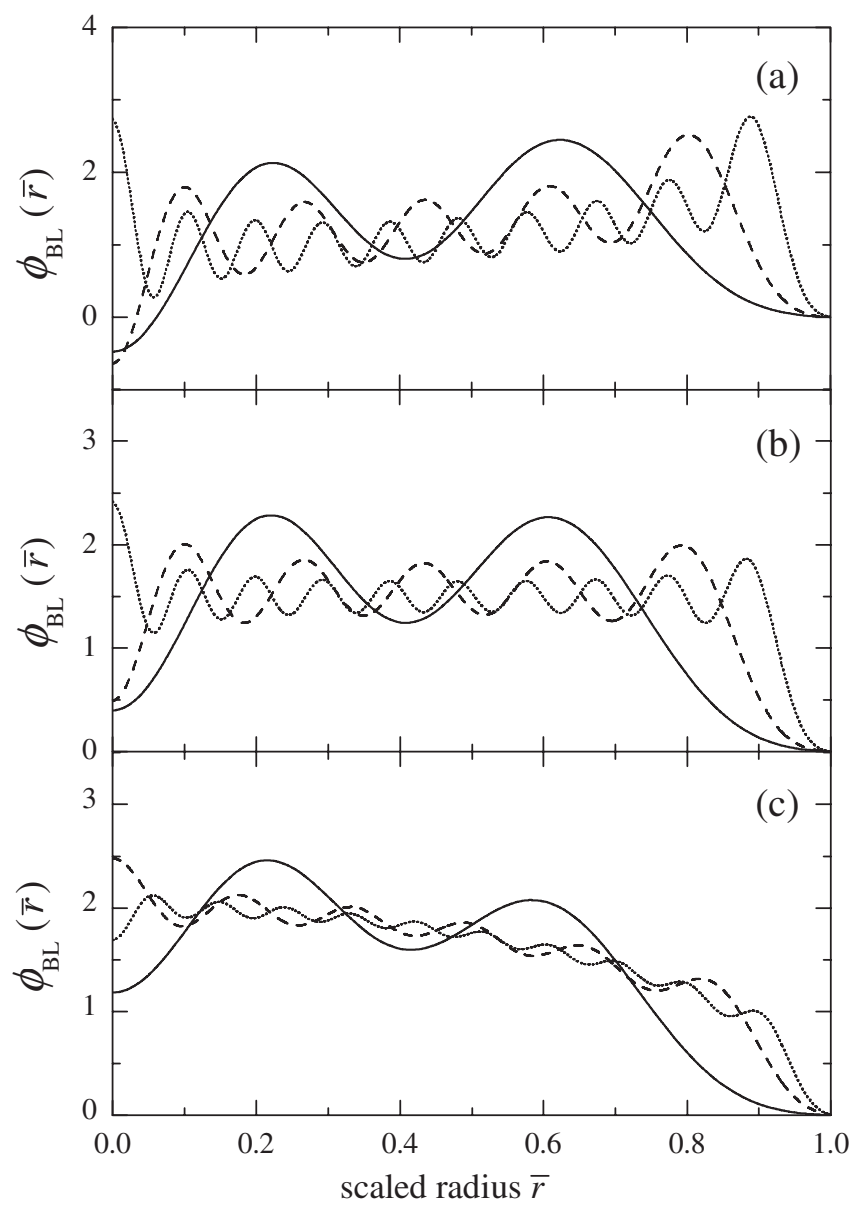

FIG. 7. As in Fig. 6, but representative band-limited beam profiles for various values of $N_{D}$. Continuous curve: $N_{D}=$ $6.77(a=12 \mathrm{~cm})$; dashed line: $N_{D}=13.58(a=17 \mathrm{~cm})$; dotted line: $N_{D}=22.74(a=22 \mathrm{~cm})$. 
Figure 7 shows the corresponding band-limited beam profiles for selected values of $N_{D}$. For the $q=0$ case (coating Brownian and thermoelastic noises), for instance, it is observed that, as $N_{D}$ increases, the profile tends to exhibit a more rapid ripple and a steepest decay. It can be argued that the flatness of the profile does not seem to be an essential ingredient for the coating noise reduction.

To sum up, it is seen that the diffraction-loss constraint sets an upper limit to the effective dimension of the optimization problem, via the finite-spatial bandwidth property of the physically admissible solutions of the cavity eigenstate equation. Thus, the only way to approach the absolute minima of the noise constituents acting on the beam/mirror profiles, under a prescribed diffraction-loss constraint, is by increasing $N_{D}$, (i.e., if the cavity length and laser wavelength are kept fixed, by increasing the mirror radius a). However, as seen from Fig. 6, increasing $N_{D}$ (or, equivalently, $a$ ) beyond a certain value pays little, as the noise curves roll off very slowly beyond a certain point, and tend to settle. Going, e.g., from $a=16 \mathrm{~cm}$ (AdvLIGO baseline design) to $a=23 \mathrm{~cm}$ reduces the coating noise only by $\sim 14 \%$. Moreover, besides the technological challenges involved, this raises some model-consistency issues related to the actual validity of the underlying ITM approximation.

\section{Validity of the ITM approximation}

In [21], the ITM approximations in (1) have been validated and calibrated against the FTM numerical solutions in [10], for GB and MB profiles. Assuming a $40 \mathrm{~kg}$ fusedsilica test mass and $\mathcal{L}_{T}=1 \mathrm{ppm}$ (design specifications for Adv-LIGO), the ITM predictions for a MB profile were found to yield errors $<10 \%$ in the coating (Brownian as well as thermoelastic) noises and $<25 \%$ in the substrate Brownian noise (the thermoelastic noise component being negligible for fused-silica test masses [10]), for mirror radii $a \lesssim 17 \mathrm{~cm}$. For sapphire test masses, the error in the (dominant) substrate thermoelastic noise component was found to be comparable to the substrate Brownian case for fused silica. Assuming that comparable figures apply to the band-limited beam profiles in (28) too, some representative values of the realistic bounds for the case $a=16 \mathrm{~cm}$ $\left(N_{D}=12.03\right)$ are reported in Table II [scaled to the corresponding absolute bounds in (22)]. One observes a moderate increase, as compared with the absolute bounds, of a factor $\sim 1.14$ for the substrate Brownian noise, $\sim 1.31$ for the coating (Brownian as well as thermoelastic) noises, and $\sim 1.55$ for the coating thermoelastic noise.

\section{COMPARISON WITH CURRENT STATUS AND TRENDS: GAUSSIAN AND MESA BEAMS}

It is suggestive to compare the above derived bounds with those attainable by the current (Gaussian) and proposed (mesa) beam profiles. For these profiles, without solving the eigenvalue problem in (11), one can exploit simple approximate analytic solutions for the dominant eigenmode, valid in the (transversely) infinite-mirror limit, estimating the relevant diffraction losses via the so-called "clipping approximation" [42], i.e., by using the infinitemirror approximate field distributions in the first equation in (16).

\section{A. Gaussian beams}

The scaled field distribution, in the infinite-radius-mirror approximation, for a GB can be expressed as

$$
\phi_{\mathrm{GB}}\left(\bar{r}, \bar{w}_{0}\right)=\Xi_{\mathrm{GB}} \exp \left(-\frac{\bar{r}^{2}}{2 \bar{w}_{0}^{2}}\right),
$$

where $\Xi_{\mathrm{GB}}$ is a normalization constant, and the waist parameter $\bar{w}_{0}$ is fixed by the clipping-approximated diffraction-loss constraint,

$$
\bar{w}_{0 c}=\left(-\log \mathcal{L}_{T}\right)^{-(1 / 2)} .
$$

In view of the particularly simple analytic expression of the field intensity distribution (and of its HT), the scaled noise functional in (10) can be computed in closed form,

$$
\begin{aligned}
\bar{S}_{\mathrm{GB}} & \equiv\left\|\bar{\kappa}^{q / 2} \mathcal{H}\left[\left|\phi_{\mathrm{GB}}\right|^{2}\right]\right\|^{2} \\
& =\frac{2^{q / 2} \Gamma\left(\frac{q}{2}+1\right)\left(-\log \mathcal{L}_{T}\right)^{(q / 2)+1}}{\left(1-\mathcal{L}_{T}\right)^{2}}, \quad q \geq-1 .
\end{aligned}
$$

\section{B. Mesa beams}

A MB profile supported by a nearly flat MH-shaped mirror can be synthesized via coherent superposition of GBs, with identical waist parameter $w_{0}$ and parallel optical axes, launched from a circular aperture of radius $R_{0}$ in the waist plane. As shown in $[15,16]$, in the infinite-mirror approximation, such a beam profile can be effectively represented in terms of a Laguerre-Gauss (LG) expansion, which, in the scaled form used here, can be written as [43]

$$
\begin{aligned}
\phi_{\mathrm{MB}}\left(\bar{r}, \bar{R}_{0}, \bar{w}_{0}, N_{D}\right)= & \Xi_{\mathrm{MB}} \exp [\imath \Theta(\bar{r})] \sum_{m=0}^{\infty} A_{m}\left(\bar{R}_{0}, \bar{w}_{0}, N_{D}\right) \\
& \times \psi_{m}\left(\frac{\sqrt{2} \bar{r}}{\bar{w}_{0} \sqrt{1+\frac{1}{\pi^{2} N_{D}^{2} \bar{w}_{0}^{4}}}}\right)
\end{aligned}
$$

In (37), $\Xi_{\mathrm{MB}}$ is a normalization constant, $\Theta(\bar{r})$ is an irrelevant phase distribution, and the expansion coefficients $A_{m}$ are given by

$$
\begin{aligned}
A_{m}\left(\bar{R}_{0}, \bar{w}_{0}, N_{D}\right)= & (-1)^{m} P\left(m+1, \frac{\bar{R}_{0}^{2}}{2 \bar{w}_{0}^{2}}\right) \\
& \times \exp \left[2 \imath m \arctan \left(\frac{1}{\pi N_{D} \bar{w}_{0}^{2}}\right)\right],
\end{aligned}
$$

with $P$ denoting an incomplete Gamma function ([26], Eq. (6.5.13)). The orthonormal LG basis functions in (37) are 


$$
\psi_{m}(\xi)=\sqrt{2} \exp \left(-\frac{\xi^{2}}{2}\right) L_{m}\left(\xi^{2}\right),
$$

with $L_{m}$ denoting an $m$ th-order Laguerre polynomial ([26], Chap. 22). In the most general case, the scaled MB profile depends on three parameters: $\bar{R}_{0}, \bar{w}_{0}$, and $N_{D}$. However, the (clipping-approximated) diffraction-loss constraint introduces a relationship $\bar{R}_{0}=\bar{R}_{0 c}\left(\bar{w}_{0}, N_{D}\right)$, illustrated in Fig. 8 for several representative values of $N_{D}$, which reduces the number of independent parameters to two $\left(\bar{w}_{0}, N_{D}\right)$. It is worth noticing that, in the topical MB literature, the waist parameter $\bar{w}_{0}$ is heuristically chosen according to a minimum-spreading criterion [44], viz.

$$
\bar{w}_{0}^{(\mathrm{MS})} \equiv \frac{1}{a} \sqrt{\frac{L}{k}}=\frac{1}{\sqrt{\pi N_{D}}},
$$

in an attempt of achieving the best tradeoff between topflatness and edge-steepness of the beam intensity profile. This further reduces the number of independent parameters to one $\left(N_{D}\right)$. When minimizing the noise functional in (17), the above choice, while intuitively sound, is not necessarily justified a priori from the mathematical viewpoint, and the more general two-parameter optimization problem

$$
\bar{S}_{\mathrm{MB}}^{(\min )}=\min _{\bar{w}_{0}, N_{D} \in \mathbb{R}}\left\|\bar{\kappa}^{q / 2} \mathcal{H}\left[\left|\phi_{\mathrm{MB}}\right|^{2}\right]\right\|^{2}
$$

should be considered instead. However, the heuristic minimum-spreading criterion in (40) is pretty close to optimal for sufficiently large $N_{D}$. As an illustrative example, the functional in (41) for $q=0$ (coating

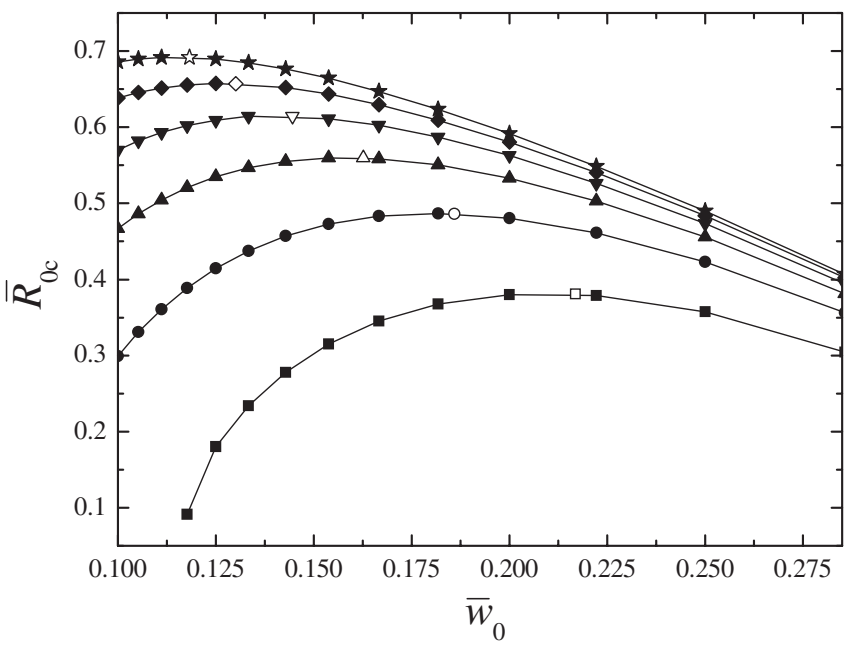

FIG. 8. Relationship between the MB parameters $\bar{R}_{0}$ and $\bar{w}_{0}$ arising from the clipping-approximated diffraction-loss constraint $\left(\mathcal{L}_{T}=1 \mathrm{ppm}\right)$, for various values of $N_{D}$ (assuming $L=$ $4 \mathrm{~km}$ and $\lambda=1064 \mathrm{~nm})$. Squares: $N_{D}=6.77(a=12 \mathrm{~cm})$; circles: $N_{D}=9.21(a=14 \mathrm{~cm})$; up-triangles: $N_{D}=12.03(a=$ $16 \mathrm{~cm})$; down-triangles: $N_{D}=15.23(a=18 \mathrm{~cm})$; diamonds: $N_{D}=18.80 \quad(a=20 \mathrm{~cm}) ;$ stars: $N_{D}=22.74 \quad(a=22 \mathrm{~cm})$. White bullets mark the minimum-spreading configurations [cf. (40)].

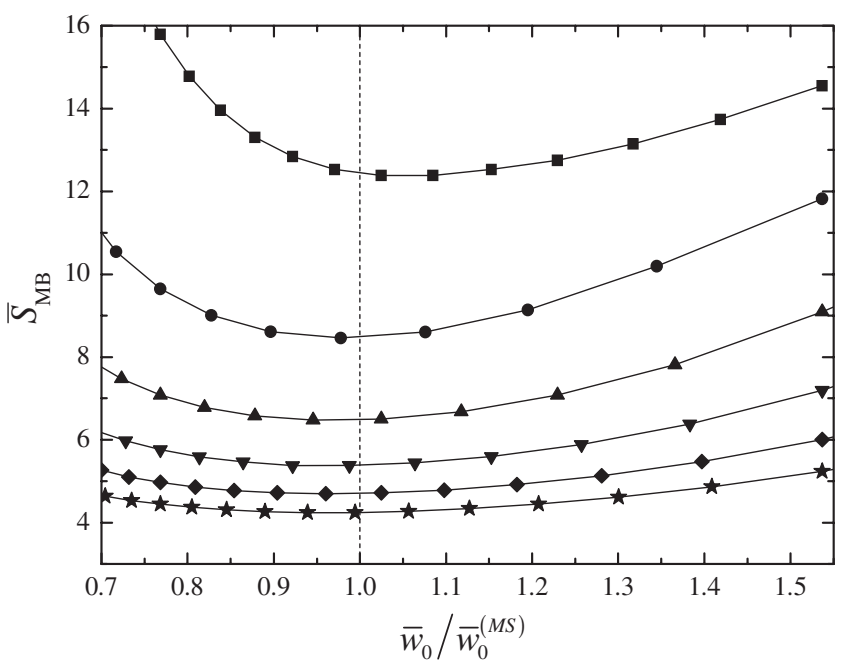

FIG. 9. As in Fig. 8, but coating $(q=0)$ noises as a function of $\bar{w}_{0}$ (scaled to its minimum-spreading value).

Brownian and thermoelastic noises) is shown in Fig. 9 as a function of $\bar{w}_{0}$ [scaled to the reference minimumspreading value in (40)], for several representative values of $N_{D}$, within the parametric range of potential interest for Adv-LIGO. It is observed that the curves at fixed $N_{D}$ exhibit a broad minimum around (but not exactly at) $\bar{w}_{0}=$ $\bar{w}_{0}^{(\mathrm{MS})}$, which becomes deeper and broader as $N_{D}$ is increased. A similar behavior is observed for the substrate Brownian $(q=-1)$ and thermoelastic $(q=1)$ noises, and is not shown for brevity.

\section{GB vs MB vs absolute and realistic bounds}

The noise levels achievable via a GB profile and a reference $\mathrm{MB}$ profile (minimum noise, for $a=16 \mathrm{~cm}$, i.e., $N_{D}=12.03$, cf. Fig. 9), scaled to the corresponding absolute and realistic bounds derived in Sec. III, are also included in Table II [45]. As already established in [10], MB profiles yield consistently lower noises than the GB counterparts, with reductions of nearly a factor $\sim 2.13$ in the coating (Brownian as well as thermoelastic) noise, and of $\sim 1.45$ and $\sim 3.07$ in the substrate Brownian and thermoelastic noise components, respectively.

By comparison with the absolute and realistic bounds, one notes a potential for significant further reductions. Specifically, as compared to the MB reference values, the realistic bounds indicate potential reductions of nearly a factor 1.8, 2.5, 2.9 for the substrate Brownian, coating (Brownian as well as thermoelastic) and substrate thermoelastic noises, respectively, thereby justifying the further exploration of alternative numerical-optimization-driven configurations.

\section{Optimal vs good profiles}

On the basis of the above analysis, a robust (e.g., genetic) optimization algorithm based on a mirror parame- 
terization consistent with the problem's effective dimension, aimed at getting as close as possible to the realistic (lower) noise bounds, could be implemented with relative ease.

It should be stressed, however, that any optimal design should also cope with some more or less obvious additional requirements to be also rated as a good design, e.g.,

(i) the optimal mirror should note pose critical technological challenges;

(ii) the optimal field should be easy to launch, i.e., should couple effectively to the injected laser beam; and

(iii) the optimal field should be reasonably stable against misalignment and mirror manufacturing tolerances.

Any candidate suboptimal designs should be ultimately gauged on the basis of their compliance with the above practical requirements.

\section{CONCLUSIONS AND PERSPECTIVES}

In this paper, based on the ITM approximations in $[20,21]$, we have addressed some key preliminary issues in connection with the optimal beam-shaping problem for thermal-noise reduction in advanced gravitational-wave interferometric detectors. The main conclusions can be summarized as follows:

(i) The estimated lower bounds in a realistic configuration, accounting for physical-feasibility-induced (diffraction losses, band limitation) and modelconsistency (ITM approximation) constraints, indicate the possibility of significant noise reductions (cf. Table II) as compared with the current status and trend. In particular, for the coating noise (dominant for the case of fused-silica test masses), a potential reduction of nearly a factor 2.5 is estimated, as compared with the MB counterpart.

(ii) The key role of the number $N_{D}$ of electromagnetic degrees of freedom (strictly related to the Fresnel number) of the optical cavity in establishing realistic lower bounds has been highlighted. In this connection, while the possibility of increasing $N_{D}$ by acting on the cavity length or the laser wavelength does not appear technologically viable for secondgeneration detectors (thereby leaving the mirror radius as the only adjustable design parameter), it could be taken into account for third-generation instruments.

(iii) From inspection of the band-limited beam profiles derived in Sec. III B 2, one can infer important (sometime counter-intuitive) prior information to intelligently drive the optimization process. For instance, for the coating noises, it clearly emerges that the flatness of the beam is not a critical requirement, since profiles with ripples (cf. Fig. 7) can perform better than flat-top MBs. This observation, which is also consistent with the results obtained using higher-order modes in spherical-mirror cavities [17], should be taken into account when parameterizing the functional space chosen for the optimization problem.

We believe that the above results pave the way for the actual optimization problem, for which a geneticalgorithm implementation (based, e.g., on an augmented Lagrangian formulation) [30] is currently under investigation. Interesting research directions include extensions of the preliminary study to higher-order (multipolar, nonaxisymmetric) modes.

While preparing this paper, we became aware of work done independently by M. Bondarescu and Y.B. Chen ([46], Chap. 3). Via a constrained-gradient-flow optimization of an LG-parameterized beam profile [27], they succeeded in retrieving a special (nearly conical) mirror profile which minimizes the coating (Brownian and thermoelastic) noise. Remarkably, their results provide a nice independent confirmation of the general conclusions drawn here. Indeed, they demonstrate the computational affordability of the optimization procedure, and confirm the key role of the mirror radius in setting the tradeoff between diffraction loss and noise reduction, in complete agreement with the general scenario outlined here. Also, the minimum noise achieved by their design gets pretty close to our corresponding realistic bound. The reader is referred to [46] for further implementation and computational details.

\section{ACKNOWLEDGMENTS}

This material is based upon work supported by the U.S. National Science Foundation under Cooperative Agreement No. PHY-0107417.

\section{APPENDIX: MINIMIZATION OF THE LAGRANGIAN FUNCTIONAL IN (20)}

The stationary solution of the variational problem obtained equating to zero the functional derivative of (20) can be easily derived using the Gateaux differential

$$
\lim _{\epsilon \rightarrow 0} \frac{\Lambda\left[f_{s}+\epsilon \delta f, \mu\right]-\Lambda\left[f_{s}, \mu\right]}{\epsilon}=0, \quad \forall \delta f \in L_{[0,1]}^{1} .
$$

It is readily verified that

$$
\begin{aligned}
\Lambda[f+\epsilon \delta f, \mu]= & \Lambda[f, \mu] \\
& +2 \epsilon\left[\left\langle\bar{\kappa}^{q / 2} \mathcal{H}_{1}[f], \bar{\kappa}^{q / 2} \mathcal{H}_{1}[\delta f]\right\rangle\right. \\
& \left.-\mu \int_{0}^{1} d \bar{r} \bar{r} \delta f(\bar{r})\right] \\
& +\epsilon^{2}\left\|\bar{\kappa}^{q / 2} \mathcal{H}_{1}[\delta f]\right\|^{2} .
\end{aligned}
$$


By using (A2) in (A1), and interchanging the spectral and spatial (HT) integrals in the inner product, the stationarity condition can be equivalently written as

$$
\int_{0}^{1} d \bar{r}\left[\int_{0}^{\infty} d \bar{\kappa} \bar{\kappa}^{q+1} J_{0}(\bar{\kappa} \bar{r}) \int_{0}^{1} d \bar{r}^{\prime} \bar{r}^{\prime} f_{s}\left(\bar{r}^{\prime}\right) J_{0}\left(\bar{\kappa} \bar{r}^{\prime}\right)-\mu\right] \bar{r}^{\prime} \delta f(\bar{r})=0, \quad \forall \delta f \in L_{[0,1]}^{1},
$$

from which it follows that the stationary profile $f_{s}$ satisfies the integral equation

$$
\int_{0}^{\infty} d \bar{\kappa} \bar{\kappa}^{q+1} J_{0}(\bar{\kappa} \bar{r}) \int_{0}^{1} d \bar{r}^{\prime} \bar{r}^{\prime} f_{s}\left(\bar{r}^{\prime}\right) J_{0}\left(\bar{\kappa} \bar{r}^{\prime}\right)=\mu, \quad 0 \leq \bar{r} \leq 1 .
$$

Equation (A4) can be solved in closed form, by inspection. Indeed, we capitalize on its nested-HT structure to use the following integral identities:

$$
\int_{0}^{\infty} \bar{\kappa}^{q / 2} J_{(q / 2)+1}(\bar{\kappa}) J_{0}(\bar{r} \bar{\kappa}) d \bar{\kappa}=2^{q / 2} \Gamma\left(\frac{q}{2}+1\right), \quad 0 \leq \bar{r} \leq 1, \quad-1 \leq q \leq 1,
$$

(see, e.g., [47], Eq. (2.12.31.1)), and

$$
\bar{\kappa}^{q / 2} J_{(q / 2)+1}(\bar{\kappa})=\frac{2^{-(q / 2)} \bar{\kappa}^{q+1}}{\Gamma\left(\frac{q}{2}+1\right)} \int_{0}^{1} d \bar{r}^{\prime} \bar{r}^{\prime}\left(1-\bar{r}^{\prime 2}\right)^{q / 2} J_{0}\left(\bar{\kappa} \bar{r}^{\prime}\right), \quad q \geq-1,
$$

(see, e.g., [47], Eq. (2.12.3.6)). By combining (A5) and (A6) one obtains

$$
\frac{2^{-q}}{\left[\Gamma\left(\frac{q}{2}+1\right)\right] 2} \int_{0}^{\infty} d \bar{\kappa} \bar{\kappa}^{q+1} J_{0}(\bar{\kappa} \bar{r}) \int_{0}^{1} d \bar{r}^{\prime} \bar{r}^{\prime}\left(1-\bar{r}^{\prime 2}\right)^{q / 2} J_{0}\left(\bar{\kappa} \bar{r}^{\prime}\right)=1, \quad 0 \leq \bar{r} \leq 1,
$$

which, by comparison with (A4), yields

$$
f_{s}(\bar{r})=\mu \frac{\left(1-\bar{r}^{2}\right)^{q / 2}}{\left[\Gamma\left(\frac{q}{2}+1\right)\right]^{2}}, \quad 0 \leq \bar{r} \leq 1 .
$$

The as yet unspecified multiplier $\mu$ in (A8) is chosen so as to satisfy the normalization constraint in (19b),

$$
\mu=2^{q}(q+2)\left[\Gamma\left(\frac{q}{2}+1\right)\right]^{2},
$$

thereby yielding the final result in (21).
From (A2) and (A4), it follows that

$$
\begin{gathered}
\bar{S}[g]-\bar{S}\left[f_{s}\right]=\left\|\bar{\kappa}^{q / 2} \mathcal{H}_{1}\left[g-f_{s}\right]\right\|^{2}, \\
\forall g \in L_{[0,1]}^{1}: \int_{0}^{1} g(\bar{r}) \bar{r} d \bar{r}=1 .
\end{gathered}
$$

Therefore, from the readily verifiable positive-definiteness of the functional norm in (A10), one concludes that the stationary profile $f_{s}$ in (21) yields the absolute minimum of the noise functional in (18).
[1] http://www.ligo.caltech.edu.

[2] S. R. Rao, Ph.D. thesis, California Institute of Technology, Pasadena, California, USA, 2003. Available online: http:// resolver.caltech.edu/caltechETD:etd-05092003-153759.

[3] http://www.ligo.mit.edu/bench/bench.html.

[4] Advanced LIGO Reference Design Report No. LIGO060056-08-M, 2007.

[5] G. M. Harry et al., Classical Quantum Gravity 24, 405 (2007).

[6] J. Agresti et al., in Advances in Thin-Film Coatings for Optical Applications III, edited by M. J. Ellison, Proc. SPIE Vol. 6286 (SPIE, Bellingham, Washington, USA, 2006).

[7] E. D'Ambrosio et al., Report No. LIGO-G000223-00-D, 2000

[8] E. D’Ambrosio et al., arXiv:gr-qc/0409075 (2004).

[9] R. O'Shaughnessy et al., arXiv:gr-qc/0409050 (2004).
[10] J. Agresti and R. DeSalvo, Report No. LIGO-T050269-00, 2005.

[11] J. Agresti et al., in Laser Beam Shaping VI, edited by F. M. Dickey and D.L. Shealy, Proc. SPIE Vol. 5876 (SPIE, Bellingham, Washington, USA, 2005).

[12] P. T. Beyersdorf et al., Appl. Opt. 45, 6723 (2006).

[13] P. Savov and S. Vyatchanin, Phys. Rev. D 74, 082002 (2006).

[14] M. Bondarescu and K. S. Thorne, Phys. Rev. D 74, 082003 (2006).

[15] V. Galdi et al., Phys. Rev. D 73, 127101 (2006).

[16] V. Galdi et al., arXiv:gr-qc/0602074 (2006).

[17] B. Mours, E. Tournefier, and J.-Y. Vinet, Classical Quantum Gravity 23, 5777 (2006).

[18] F. Bondu, P. Hello, and J.-Y. Vinet, Phys. Lett. A 246, 227 (1998).

[19] Y.T. Liu and K.S. Thorne, Phys. Rev. D 62, 122002 
(2000).

[20] R. O’Shaughnessy, Classical Quantum Gravity 23, 7627 (2006).

[21] G. Lovelace, Classical Quantum Gravity 24, 4491 (2007).

[22] Y. Levin, Phys. Rev. D 57, 659 (1998).

[23] O. M. Bucci and G. Franceschetti, IEEE Trans. Antennas Propag. 37, 918 (1989).

[24] J. Agresti et al., arXiv:gr-qc/0511062 (2005).

[25] This investigation does not deal with tilt (in)stability issues, for which nearly flat and nearly concentric configurations exhibit significantly different responses [13].

[26] M. Abramowitz and I. A. Stegun, Handbook of Mathematical Functions (Dover, New York, 1964).

[27] A.E. Siegman, Lasers (University Science Books, Mill Valley, California, 1986).

[28] The concept of electromagnetic degrees of freedom [23] is related to the minimum number of basis functions that are needed to represent within a bounded region, with a prescribed error in a given metric, an electromagnetic field produced by finite-support (impressed or equivalent) sources.

[29] P. Pedregal, Introduction to Optimization (SpringerVerlag, New York, 2004).

[30] Electromagnetic Optimization by Genetic Algorithms, edited by Y. Rahmat-Samii and E. Michielssen (Wiley, New York, 1999).

[31] A. Hoorfar, IEEE Trans. Antennas Propag. 55, 523 (2007).

[32] J. Robinson and Y. Rahmat-Samii, IEEE Trans. Antennas Propag. 52, 397 (2004).

[33] D. R. Smith, Variational Methods in Optimization (Dover, New York, 1998).

[34] The satisfaction of the positivity constraint in (19a), not explicitly enforced, is verified a posteriori.

[35] D. Slepian, Bell Syst. Tech. J. 43, 3009 (1964).

[36] H. Xiao, V. Rokhlin, and N. Yarvin, Inverse Probl. 17, 805 (2001).

[37] Y. Shkolnisky, Appl. Comput. Harmon. Anal. 22, 235 (2007).
[38] Throughout the paper, this more generic denomination will be utilized, instead of the more detailed "PSWFs on a disc" in [37].

[39] The PSWF basis is the spectrum of the eigenproblem in (11) with $V=0$, representing the eigenmodes of a confocal spherical Fabry-Perot cavity with finite-size mirrors.

[40] In our approach, based on the PSWF computation via the polynomial expansions in [37], the scalar products in (29) can be efficiently computed in a semianalytic fashion.

[41] In view of the inherent discreteness of the problem, it was not always possible to find values of MT so that the diffraction-loss constraint was tightly satisfied (i.e., diffraction loss smaller but close to the targeted value). In order to ensure the comparison among configurations yielding comparable diffraction losses, we slightly relaxed the constraint, looking for values of MT yielding diffraction losses closest $(\sim \pm 0.3 \mathrm{ppm})$ to the targeted value.

[42] E. D’Ambrosio, Phys. Rev. D 67, 102004 (2003).

[43] A similar LG expansion was also derived for analytic parameterization of the $\mathrm{MH}$-shaped mirror-deformation profile [cf. [16], Eq. (34)].

[44] The Rayleigh distance of the GBs launched is chosen as coincident with the distance from the aperture plane to the mirror.

[45] Note that, although the GB and MB results in Table II have been obtained, for computational convenience, using the infinite-mirror approximation in (37), they were checked to agree to within $15 \%$ with those obtained from the numerical solution (via the Nystrom method [48]) of the eigenproblem in (11), for a number of representative cases.

[46] M. Bondarescu, Ph.D. thesis, California Institute of Technology, Pasadena, California, USA, 2007. Available online: http://resolver.caltech.edu/CaltechETD:etd05282007-231321.

[47] A.P. Prudnikov, Y. A. Brychkov, and O.I. Marichev, Special Functions, Integrals and Series Vol. 2 (Gordon \& Breach, New York, 1986).

[48] L. F. Canino et al., J. Comput. Phys. 146, 627 (1998). 